\title{
Curated Generative Fashion Design
}

\author{
Fredrick Shaap* \\ Qiueue Research, Texas, USA
}

*Corresponding author: Fredrick Shaap, Qiueue Research, 401 Congress Ave. Austin, Texas 78701, USA.

Received Date: July 16, 2020

Published Date: July 24, 2020

\begin{abstract}
The relevance of generative algorithmic design to fashion use cases has been demonstrated by way of an example involving the design of a fashion accessory item. The concept was used for designing the hardware for a modern interpretation of a woman's vintage cinch belt with an interlocking buckle. The design variants made from a beta titanium alloy for minimum weight and maximum stiffness were generated and curated to an optimal subset for volume manufacturing.
\end{abstract}

Keywords: Generative computer-aided design; Algorithmic fashion design; Aesthetic enhancement; Parametric design program

\section{Introduction}

The author has previously published examples of generative algorithmic design used for fashion design [1,2]. Similar to its applications in industrial design, engineering design and architectural design, it was shown that generative computer-aided design (GCAD) can be utilized to create fashion design variants encompassing attributes which readily meet and exceed the technical and aesthetic requirements of the task. In [1], using a women's footwear design project as an example, it was shown that a standard GCAD-based workflow can systematically create fashion design solutions which can be curated by the designer to result in optimal variants for volume production. In [2], the same workflow was employed to create design variants for a women's cuff braceletstyle smart watch, for which the sterling silver bracelet featured geometric perforations for the dual purpose of jewelry weight reduction as well as aesthetic enhancement.

\section{Tools}

A wide variety of GCAD application software products are currently available for producing generative algorithmic design solutions developed specifically for use cases in architecture, construction, engineering, etc. These products are either of the more specialized and advanced type which are typically provided as standalone application software, or they are of the generic type which are included as a module within a CAD software package. At the time of this writing, to the best of author's knowledge, there are no fashion-specific GCAD software products commercially available, either in the standalone or the modular format for practical use cases. However, the flexibility offered through selective scripting provides the ability to customize and optimize the relevant end-user programs for fashion design applications. As an alternative, fashion-specific design application software can be used in conjunction with parametric design programs to develop an integrated fashion-specific GCAD tool for fashion designers.

\section{Project}

In this use case, GCAD was utilized to produce algorithmic design variants for a modern interpretation of a women's vintage cinch belt with an interlocking buckle to be made from Ti-6-4, a beta titanium alloy. As a popular fashion accessory, cinch belts highlight 
the waistline and enhance the silhouette of the dresses featuring a relaxed fit. As an example, from the online retailer JasGood $\AA$ and its curated fashion accessory collection, Figure 1 shows a cinch belt worn over a relaxed-fit silk blouse. Adapted from the same catalog listing, the figure shows the details of this belt including the front and the back of its interlocking buckle mounted on a leather-tabbed wide elastic strap using a triple screw set at each end.

Figure 1: Cinch belt retailed by JasGood [JasGood®].

Following the design workflow described previously [1], an initial hand sketch of the belt hardware was digitized and scaled in preparation for the GCAD project. The pattern was further edited and refined using a fashion-specific application software. It was decided to design each interlocking half of the buckle as two separate components, a decorative base plate, and a load-bearing attachment. Shown in Figure 2, the decorative base plate consists of multiple partial metal discs decorated on its face by an abstract floral set. On the back-side, it has a set of three counterbores for attaching to the belt's leather-tabbed elastic strap sewn in at each end of the belt.

Figure 2: Decorative base plate.
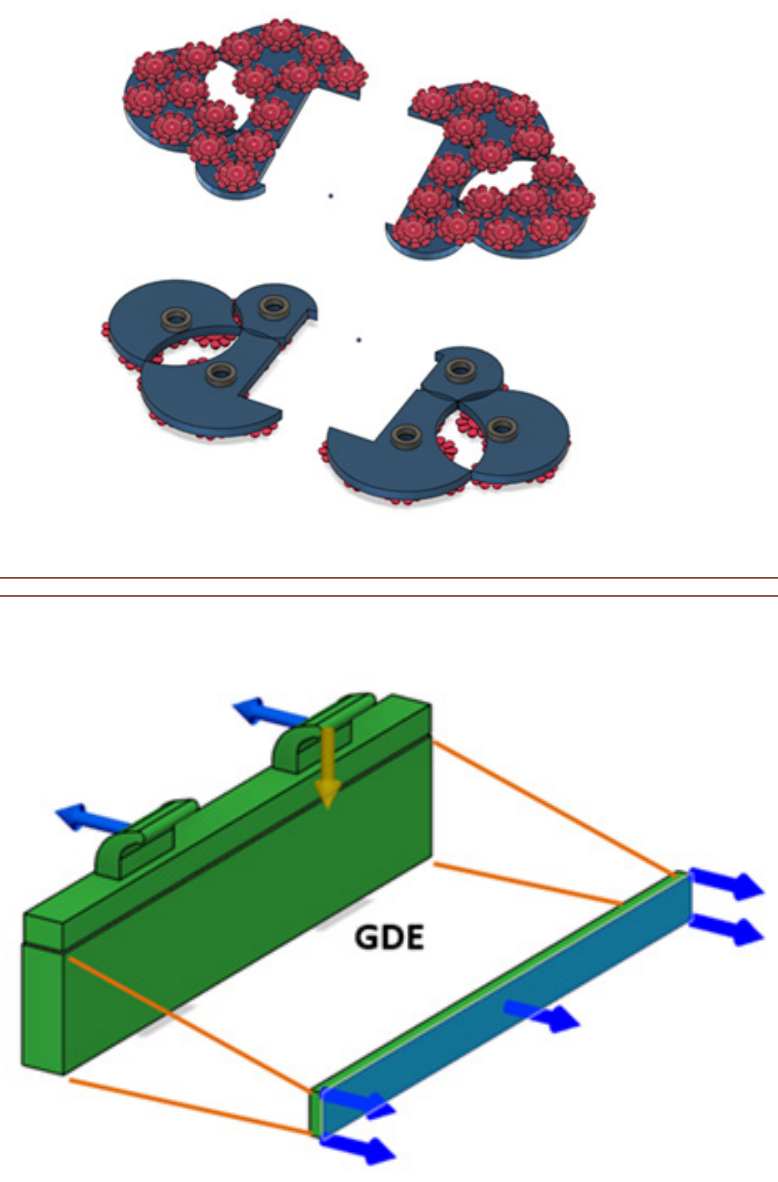

Figure 3: Load-bearing attachment and tensile forces to be absorbed. 
Figure 3 shows the load-bearing attachment, which consists of a narrow side-bar for attaching to the decorative base plate, and a wide side-bar including the interlocking mechanism at its top edge. As shown in the figure, the empty space between the narrow sidebar and the wide side-bar specified the generative design envelope (GDE) for integrating all three parts of the load-bearing attachment. This figure also shows the tensile forces and the resulting bending moment to be absorbed by the load-bearing attachment without resulting in any measurable elastic deformation and no plastic deformation and/or risk of fracture.

The GCAD requirements for generating variants of the above component in multiple iterations included: a) minimum weight; b) maximum stiffness; and c) meeting the restrictions of the selected manufacturing method. A generated design within the GDE in its raw format is shown in Figure 4. Such raw GCAD solutions were edited and refined for their manufacturing-ready design version following the below general guideline. Each tentacle-like extremity (TLE) attaching the narrow side-bar to the wide side-bar within the GDE can be replaced with a larger TLE along the same long axis. The rationale is based on the argument that a load-bearing TLE can always be replaced with one of the higher structural strength. Such replacement not only improves the mechanical stability of the hardware, but also can enhance its aesthetic appeal. For example, Figure 5 presents the edited and refined version of the GCAD solution shown in Figure 4.

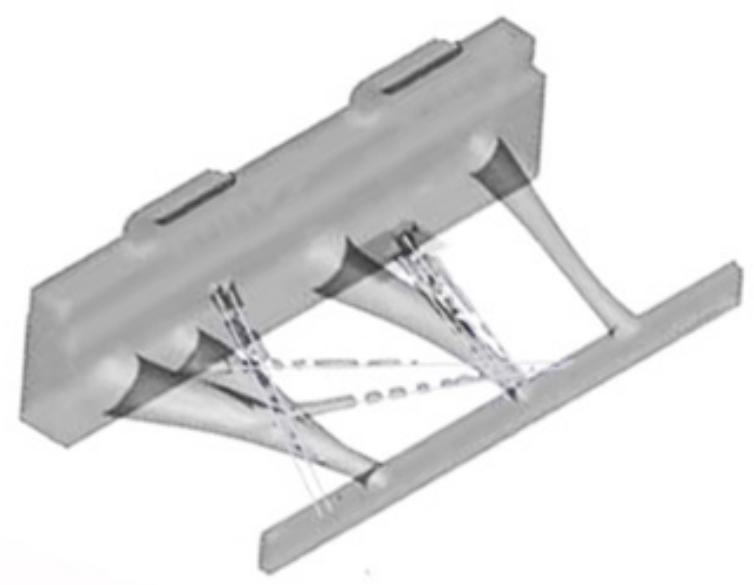

Figure 4: Example of GCAD-generated insert within GDE.

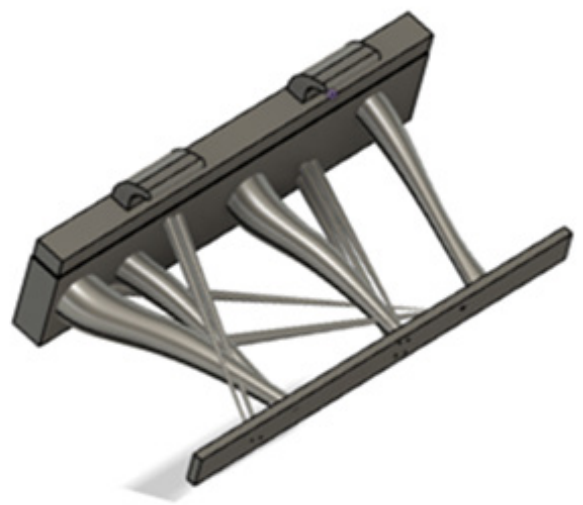

Figure 5: Edited and refined version of GCAD-generated insert shown in Figure 4.

Figure 6 shows a curated set of four cinch belt hardware designs in which the GCAD-generated TLE elements contribute both to the structural as well as the aesthetic aspects of the design. This subset of belt hardware designs, including the GCAD-generated insert configuration was selected by the fashion designer based on aesthetic appeal and range of visual dissimilarity. Figure 7 shows the renderings of hardware designs shown in Figure 6, and in Figure 8, a close-up of the interlocking hook and half-loop fastener is shown. 


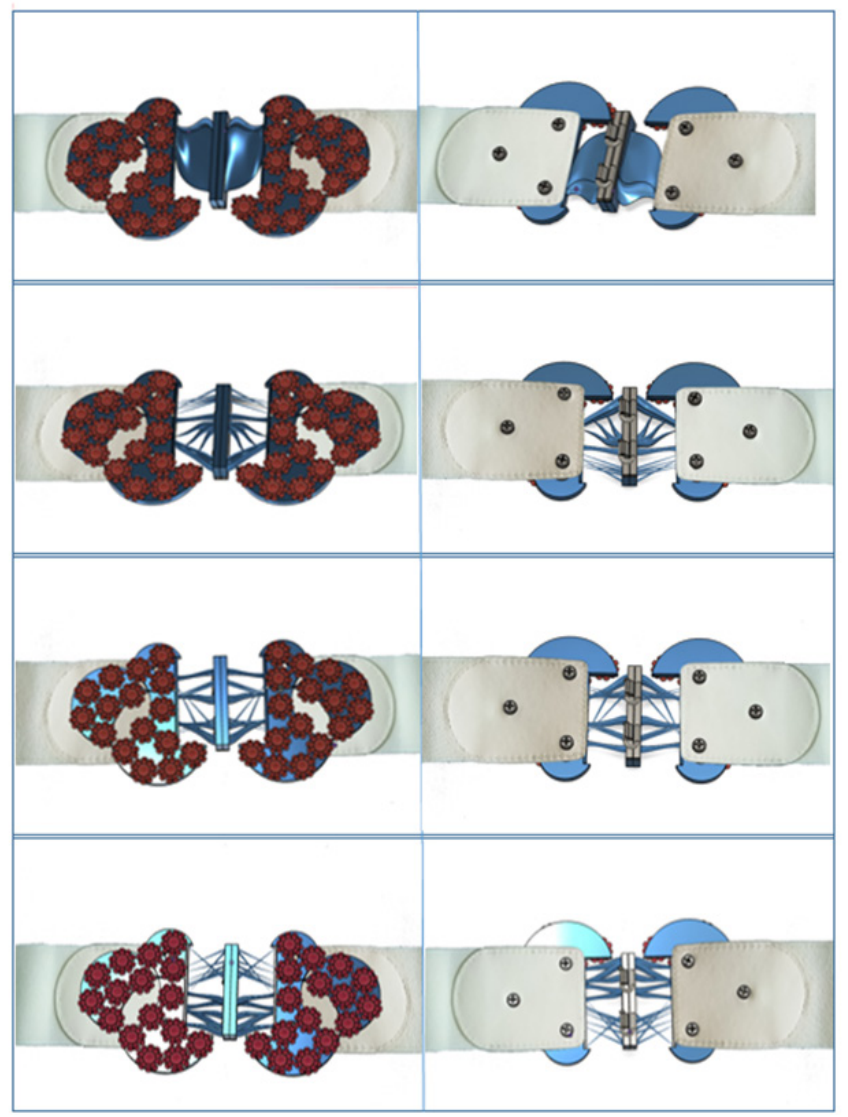

Figure 6: Curated set of partially GCAD-generated hardware designs.

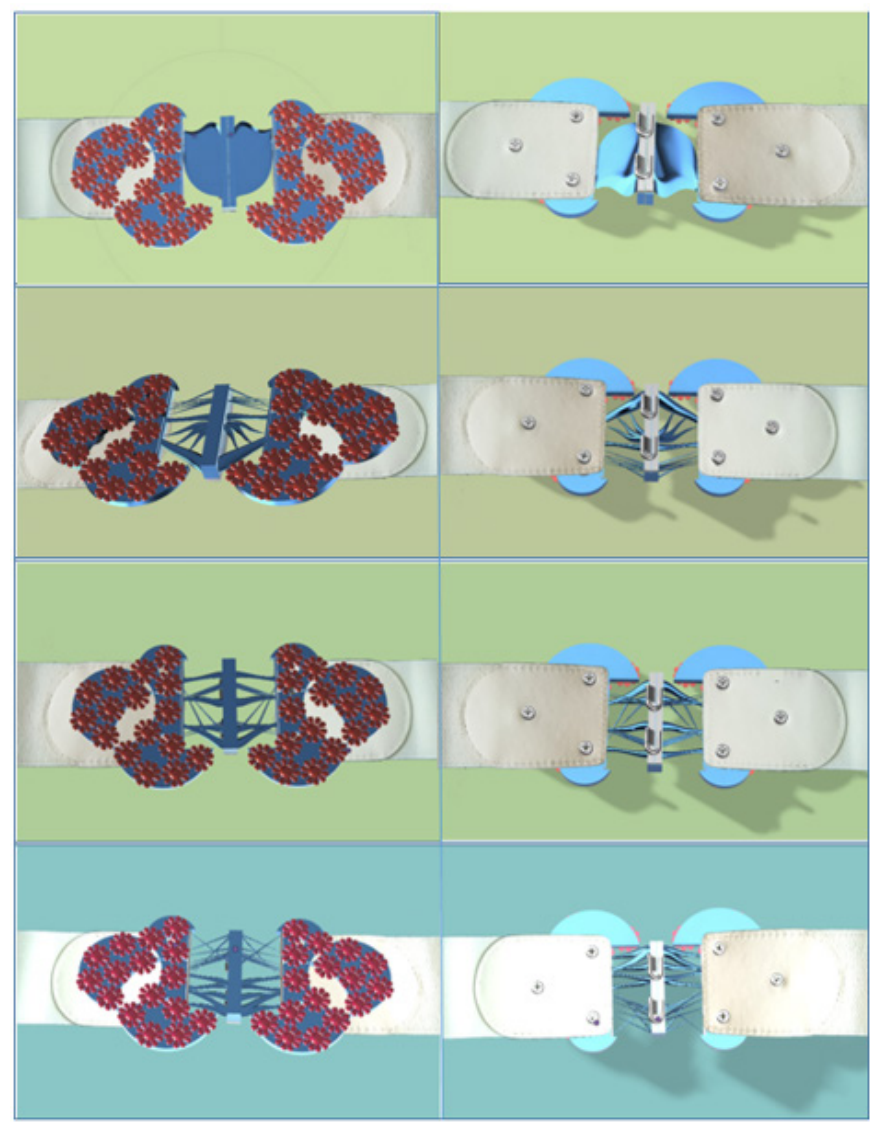

Figure 7: Rendering of designs shown in Figure 6. 
Figure 8: Details of interlocking fastener.

\section{Conclusion}

GCAD should be considered and added as a new application software tool to the fashion designer's digital tool set for enhancing both the structural as well as the aesthetic aspects of design.

\section{Acknowledgement}

None.

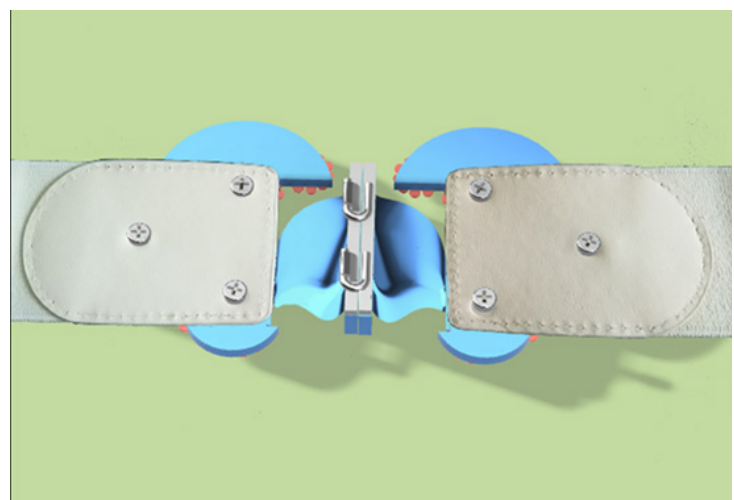

\section{Conflict of Interest}

None.

\section{References}

1. Shaap F (2020) Generative Computer-aided Fashion Design. Curr Trends in Fashion Technol Textile Eng 6(4): 1-4.

2. Shaap F (2020) Generative Algorithmic Fashion Design. Int J of Fashion Technol Textile Eng 3(1): 1-7 